\title{
On the Advertising of an Event: A Mathematical Approach
}

\author{
Rosa Ferrentino*, Carmine Boniello \\ Department of Economic and Statistics Sciences, University of Salerno, Fisciano (Salerno), Italy \\ Email: ^rferrentino@unisa.it, cboniello@unisa.it
}

How to cite this paper: Ferrentino, R. and Boniello, C. (2017) On the Advertising of an Event: A Mathematical Approach. Applied Mathematics, 8, 1648-1661. https://doi.org/10.4236/am.2017.811119

Received: October 29, 2017

Accepted: November 25, 2017

Published: November 28, 2017

Copyright $\odot 2017$ by authors and Scientific Research Publishing Inc. This work is licensed under the Creative Commons Attribution International License (CC BY 4.0).

http://creativecommons.org/licenses/by/4.0/

\section{c) (i) Open Access}

\begin{abstract}
The present contribution arises with the intent of providing a brief, and therefore not exhaustive, theoretical accommodation of the problematics related to the programming and the achievement of an event. In reality, the aim of the paper is to develop optimal advertising policies for an event that takes place at a given future instant of time (a sport game, a concert, a ballet or a theatre performance) and to minimize the advertising costs concerning the organization of the event. More precisely, the paper faces the problem of how to diffuse over time the informations about a forthcoming event among a population of potential participants and it takes into consideration, among the main vehicles for the dissemination of information, the word-of-mouth effect, a kind of advertising which is independent of the firm advertising policy.
\end{abstract}

\section{Keywords}

Advertising, Event, Word of Mouth, Goodwill

\section{Introduction and Research Aims}

By some time, it is increasingly evident the growth, both domestically and internationally, of the importance of events such as a concert, a theatre performance, a ballet or a sport game, namely events, and not necessarily sporting, that reach large shares of spectators overcoming geographical barriers.

The dissemination of informations on an event (or on a product, on a service, on an idea), within a society or within a group of individuals, can be done through a variety of means as, for examples, all types of media, of promotional activities, of public relations, and so forth, but can be done also through all communication activities that are not controlled directly by the organizers as the word-of-mouth processes and the newspaper articles. Among main vehicles for 
the dissemination of information about an event this paper focuses on the advertising implemented by the word-of-mouth.

The topic dealt in this paper is one of the themes to which many researches present in the literature have placed more attention and the growing interest towards this topic has contributed, over the past decades, to the development of several methods. In other words, some important papers on the subject and some relevant contributions are present in literature. The paper that initiated this stream of research is Robinson and Lakhani [1].

For other contributions to this area, it is possible see Feichtinger, Hartl, and Sethi, Ferrentino and Boniello, Jørgensen and Zaccour, [2] [3] [4]; these last use often the differential game theory to characterize advertising strategies. Optimal advertising strategies, relying on word-of-mouth, have been studied also by Ozga, Gould, Sethi [5] [6] [7] [8]. These papers, however, use different dynamics compared to those used in this paper. Furthermore, Teng and Thompson, Dockner and Jørgensen [9] [10] [11] and Horsky and Mate [12] studied advertising policies in a generalized Bass model, with word-of-mouth effect. A related problem is studied by Buratto and Grosset [13] who, however, do not include the word-of-mouth that have deepened papers of other authors and have suitably indicated quotations and paraphrases.

In this paper the authors study how to inform a population of potential participants about a fourth coming event and how to minimize the advertising costs concerning the organization of a particular event. In other words, the aim of this paper is to develop optimal advertising strategies to program the advertising campaign for an event that takes place at a given future instant of time and to minimize, in general, the costs of advertising for the its organization.

The sale of the tickets is driven by advertising implemented by the organizers (who can use a variety of communication tools: internet, print media, TV channels etc.) as well as by the word-of-mouth effect and this paper, among the main vehicles or instruments for the dissemination of information about the considered event, focuses on the advertising obtained with the word-of-mouth. The attention, namely, is focused on the word-of-mouth communication, where the knowledge about the event is transmitted from informed individuals to non-informed individuals and there is not a cost for the organizers: this type of advertising is free. In other words, the authors take into consideration above all the word-of-mouth effect but, anyway, it is also highlighted, for the success of the event, the role, in all its forms, of the other advertising channels.

Another objective considered in this paper is the problem of determining the advertising policy to prepare the introduction of a new product in the market and the planning of a pre-launch publicity campaign. The advertising activity has an important role in the introduction of a new product: just think, for instance, at the car industry, where an advertising campaign of sensitization usually precedes the distribution of a new car model.

The effect of advertising on sales is mediated by a state variable, called good- 
will, introduced by Nerlove and Arrow [14], that summarizes the effects of current and past advertising outlays on demand or better represents the product image by the consumers and whose evolution is described by a linear differential equation. Many papers are based on the goodwill concept of Nerlove and Arrow, possibly with different interpretation; in fact, the goodwill variable may assume different meanings, depending on the particular context. Consequently, the aim of this paper is the resolution of a typical problem in the firm theory and, namely, to minimize the total advertising costs and to maximize the expected utility coming from the goodwill level at the launch time $T$. The problem that comes out is a problem of optimal control and the number of seats sold and the advertising effort of the organizers are, respectively, the state and control variables; this problem is solved by using the stochastic control theory and by exploiting some recent results of the stochastic linear quadratic control theory. In fact, many marketing policies can be correctly explained and analyzed only by a stochastic approach to the problem. To achieve this, is built a diffusion model where the organizers control the advertising expenditures and look for to maximize the profits.

Though not exhaustive, the major contribution of this paper is that it provides a general mathematical framework for assessing the minimization of the costs of advertising of an event and that it has a context less specific compared to discussion, on the advertising policies, of papers present in literature.

The paper is organized as follows. In Section 1, after the introduction in which is analyzed the current research focus on the theme, the authors present the aim of the paper while in Section 2 they search to define the advertising communication and to describe briefly how a well-planned communication program must assure that the revenue from the sold tickets fully cover all the costs. In Section 3 and 4, instead, is formally introduced the substantial part, the plan of this paper; are, namely, provided some important modelling approaches present in literature and adopted to estimate and to minimize the costs of advertising of an event and to discuss the problem of determining the advertising policy for the introduction of a new product into a market. Section 5, finally, provides a conclusion and contains a general discussion on directions for future research: the authors hope, in fact, in the growth of research in this area. In others words, the article ends with some concluding remarks while the last part of this article represents the reviewed references.

\section{Advertising Communication}

The event is, by definition, a happening or an initiative of particular importance which must have a limited duration in the time and must satisfy some needs, namely it must have a specific finality. Shone and Parry [15], who have dealt with this theme, define the event as "that phenomenon arising from those non-routine occasions which have leisure, cultural, personal or organizational objectives set apart from the normal activity of daily life, whose purpose is to en- 
lighten, to celebrate, entertain or to challenge the experience of a group of a people".

The figures that a different title may be involved and interested in the event can be numerous and have considerably different characteristics. All of this, highlights the complexity of the organizing an event and justifies the demand, for the organizers, of an adequate professionality, namely, of skills needed to develop functions that are not simply technical functions but also managerial, in the widest sense of the term.

Nerlove and Arrow [14] assume that the demand of an event and the effect of advertising on sales depend on a state variable, called goodwill, which represents the effects of the firm investment in advertising. In reality, the goodwill is a state variable that represents the image of organizing company in the market or better summarizes the advertising investments of the organizing company and it influences the demand of the product together with price and other external factors. The effects of the advertising policy on the sales until the time $T$ depend on the goodwil value at time $\mathrm{T}$ : the greater the goodwill value at $\mathrm{T}$, the higher the following sale rate. Moreover, Nerlove and Arrow assume that the goodwill is created and increased by the event advertising, possibly by using different channels. The goodwill is, therefore, seen as a stock of productive capital subject to depreciation, namely declines spontaneous proportional to its value and, on the other hand, it is sustained by the investment flow controlled by the firm. In other words, through the flow of advertising or other forms communication, it is possible control the evolution of goodwill in the programming interval $[0, T]$. The advertising communication, in fact, contributes to increase the level of goodwill that, otherwise, without a direct action, is subject to a spontaneous decline. There are two different kinds of advertising channels. The first concerns the goodwill evolution in a deterministic way (which is called deterministic channel): the advertising message is correctly understood by the consumers and contributes to increase the goodwill value or to slow down a goodwill decrease. The second (which is called the stochastic channel) has a double effect: it surely increases, or slows down a decrease of the goodwill expectation, but it also introduces some uncertainty in the goodwill evolution. The stochastic channel advertising message has an unforeseeable effect on the goodwill, because either it may not be completely understood by the consumers, or, on the contrary, it may meet the taste of the public very well.

Then, one of the objectives of the organizers is to control the goodwill evolution in order to obtain a demand as close as possible to the congestion threshold. In fact, a lower level of the threshold means that some tickets have not been sold and, therefore, that the revenue is less than the potential maximum one. On the contrary, a higher level of the threshold would signify that the demand is greater than the offer; in this case would exist some unsatisfied customers who cause an unfavorable word-of-mouth publicity, with a negative effect on the success of future events. Instead, the organizers must take into account the customer satis- 
faction in order to obtain a well-organized event.

The organizers must, first of all, fix the date of the event date and, subsequently, the one in which the pre sales close to know in advance the number of all unsold tickets to be placed on the market by using new ways, for example by offering the packages of tickets unsold at a more affordable price than the pre sale. In fact, once a show has been scheduled to take place in certain place at a given date, the supply capacity is fixed. Consequently, unsold tickets are lost and any demand exceeding the capacity cannot be backlogged. This means that nothing happens after the event has taken place and hence, naturally, the objective of the dynamic optimization model would not include a salvage value. Therefore, a well-planned communication program should assure that the revenue from the sold tickets fully cover all the costs.

The a priori estimate of the event is of crucial importance since it allows to estimate, also if in forecast, the alleged revenues that can be obtained and which are the result of the sale of tickets and allows to be able to make organizational decisions, logistics etc.

In conclusion, to be able to reach the majority of all possible subjects, it is need to use all the various channels of communication available or better to use several communication channels that can be divided into two classes: the first includes all means of communication before described and which are directly controlled by the organizers while the second includes those communication activities that are not directly controlled by the organizers. In this latter category there is the verbal communication (the word-of-mouth) that can definitely be considered as an excellent communication tool as it is able to emotionally involve the supposed beneficiaries of the event. According Godes \& Mayzlin [16], the word-of-mouth communication "is often an important driver of consumer buying behavior such as the adoption of a new technology, the decision to watch a TV show, or the choice of which laptop to purchase".

The problem is solved formulating a model that describes the situation and using the theory of stochastic control, in particular, a cost function and a quadratic penalty function. The results obtained allow to compare the effectiveness of advertising channels and to establish how the risk connected with the channel stochastic influences its use; besides, even if the cost of the channel stochastic become null, the problem continues to be well placed because the risk associated with the action of the decision can be interpreted as a additional cost.

The presence of the stochastic control makes the problem mathematically interesting and different from others. For the generality of the problem it is necessary to study some sub-problems which are both simple from a mathematical point of view and relevant for their economic features.

\section{An Optimal Advertising}

In this section, the authors adopt a diffusion model where the organizers control their advertising expenditures and wish to maximize profit or better they consider a typical optimal control problem where an advertising intensity is looked 
for to optimize a suitable firm objective. The main objective is, then, to determine, using optimal control techniques, an optimal price or advertising rate over time.

The optimal advertising policy, besides being dependent on the cost and revenue, is also affected by the length of the planning period and by the relation between the number of seats and the total number of potential attendees. To this end, the authors consider an organization that has planned a social event (a concert, a workshop, a football), at a fixed time $T$, in a place with a limited number of seats and they suppose that, in order to stimulate as wide a participation as possible, has been organized an advertising campaign, that the customers pay the tickets only at time $T$ and that the demand depends on the event goodwill at $T$. Furthermore, they take into consideration a word-of-mouth effect (this means that who buys a ticket talks about it to its friends), and therefore is unnecessary to inform the people with other advertising: newspapers, radio and television. Such advertising is, furthermore, costly. Instead, the word-of-mouth advertising, with which individuals who are aware of the event forward information about the event to individuals who are unaware, is a type of advertising free.

In the model that the authors present, the time $t$ is a continuous variable and the planning period is equal to interval $[0, T]$ where $T$ represent the instant of time in which the event will take place; $T$ is, therefore, fixed and unmovable [1].

Let $P(t)$ be the fraction of the number of potential attendees that have bought a ticket by time $t$. The time-derivate of $P(t)$, henceforth denoted by $P^{\prime}(t)$, represents the sales rate at time $t$ and satisfies the following equation:

$$
P^{\prime}(t)=\beta a(t)[1-P(t)]+\gamma P(t)[1-P(t)] \text { with } P(0)=0
$$

where:

$a(t)$ is the rate of advertising effort used by the organizers

$\beta$ represents the efficiency of the advertising of the organizers

$\gamma$ represents the efficiency of the word-of-mouth communication.

The parameters $\beta(\beta>0)$ and $\gamma(\gamma \geq 0)$ are time-independent which is a plausible assumption if the time horizon, as here, is short.

Putting in evidence $[1-P(t)]$, the (1) becomes:

$$
P^{\prime}(t)=[1-P(t)][\beta a(t)+\gamma P(t)]
$$

$P(t)$ is a nondecreasing function of time and is upper delimited by one, which represents the normalized number of potential participants in the event. As $P(t)$ tends to one, the effect of the advertising decreases, because the number of participants in the concert is predetermined, as established by a careful market survey, performed before every organizational decision, from which it emerges the forecast of the number of potential attendees.

Since the number of seats is fixed and the number of potential attendees is normalized to 1 , it is need to consider the state constraint $P(t) \leq r$ where $r$ is the fraction of the number of potential attendees that can sit down and 
$t \in[0, T]$. This is a plausible assumption, unless the stadium or concert hall or theater is very large and the event is utterly uninteresting.

Of course $r<1$, that is, the number of seats does not exceed the number of potential attendees.

Therefore, the objective organizers are to minimize:

1) The advertising costs

2) The number of unsold seats on the day the event takes place

In the particular case that is presented here, the advertising efforts are costly; consequently, the advertising costs are represented by a quadratic and convex function $C(a)$ and this is mainly a matter of mathematical convenience and is consistent with the choice done.

Then, $C(a)$ represents the total costs supported for advertising and it is equal to:

$$
C(a)=v a+\frac{w}{2} a^{2}
$$

where:

$v \geq 0$ and $w>0$ are constants. For $v>0 \quad C^{\prime}(0)$ is positive.

The implication is that, for a sufficiently large value of $v$, it can not pay to advertise at all, but there is a need to make informed choices. In fact, if this is true, no tickets will be sold at all without distinction because $P(t)$ is equal to zero. The value of $w$ determines the magnitude of the advertising rate whenever it is positive. Therefore, higher will be the value of $w$, smaller will be the advertising rate.

Consequently, the function that must be minimized is:

$$
f(t)=r+C(t)-P(t)
$$

where:

$$
C(t)=\int_{0}^{T} C(a) \mathrm{d} t
$$

namely

$$
C(t)=\int_{0}^{T}\left[v a(t)+\frac{w}{2}(a(t))^{2}\right] \mathrm{d} t,
$$

is the total advertising cost incurred while $P(t)$ is:

$$
P(T)=\int_{0}^{T} P^{\prime}(t) \mathrm{d} t
$$

namely

$$
P(T)=\int_{0}^{T}\{[\beta a(t)+\gamma P(t)][1-P(t)]\} \mathrm{d} t
$$

Therefore, the function to minimize becomes:

$$
f(t)=r+\int_{0}^{T}\left[v a(t)+\frac{w}{2}(a(t))^{2}\right] \mathrm{d} t-\int_{0}^{T}\{[\beta a(t)+\gamma P(t)][1-P(t)]\} \mathrm{d} t
$$

or better: 


$$
f(t)=r+\int_{0}^{T}\left\{v a(t)+\frac{w}{2}(a(t))^{2}-[\beta a(t)+\gamma P(t)][1-P(t)]\right\} \mathrm{d} t
$$

The problem that comes out is a problem of optimal control and the number of seats sold and the advertising effort of the organizers are, respectively, the state and control variables; this problem can be solved by using the stochastic control theory; more precisely, the optimal control problem can be solved using either the Pontryagin Maximum Principle or the Hamilton Jacobi Bellman Equation approach (the first leads to open-loop strategies which only depend on time while the second leads to closed-loop strategies which depend also on the state variables). To this end must be defined the Hamiltonian function (omitting for simplicity the time argument $t$ ):

$$
H=(P, a, \lambda)=\left(\lambda_{0}+\lambda\right)(\beta a+\gamma P)(1-P)+\lambda_{0}\left[v a+\frac{w}{2} a^{2}\right]
$$

Assuming the existence of an optimal solution, the Maximum Principle provides the necessary conditions of optimality (Seierrstad \& Sydsaeter [17]).

Exists, thus, a constant $\lambda_{0}$ and a differential function $\lambda(t)$ such that for each $t \in[0, T]$ it holds that $\left(\lambda_{0}, \lambda(t) \neq(0,0)\right)$. Consequently, the Lagrangian:

$$
L=H+\eta a=(1+\lambda)(\beta a+\gamma P)(1-P)+\left[v a+\frac{w}{2} a^{2}\right]+\eta a
$$

provides the following conditions of optimality (omitting for simplicity the time argument $t$ ):

$$
\begin{gathered}
\frac{\partial L}{\partial a}=(1+\lambda) \beta(1-P)-v-w a+\eta=0 \\
\lambda^{\prime}=[1+\lambda][\beta a-\gamma(1-2 P)] \\
a \geq 0, \eta a=0, \eta \geq 0 \\
\lambda(T) \leq 0, \lambda(T)[P(T)-r]=0, P(T) \leq r
\end{gathered}
$$

It is readily shown that $\partial^{2} H / \partial a^{2}=-w<0$. Hence the Hamiltonian is strictly concave in $a$ and the maximization yields a unique control $a$.

Differentiating in (10) with respect to time, and using (1), (11), and (12) it is possible get

$$
a^{\prime}=-\frac{\gamma}{w}[1-P][v+w a]
$$

which is negative.

Thus, the advertising efforts are decreasing over time. In fact, from (14) readily follows $\partial^{2} a / \partial t^{2}>0$ which means that advertising efforts decrease over time with an increasing rate.

This result is due to the word-of-mouth effect; the advertising is used to generate initial awareness of the event, but as soon as the word-of-mouth process gains momentum, there is less need for advertising.

In the case in which there is not a word-of -mouth effect (therefore $\gamma=0$ ) the number of tickets sold, $P(t)$, can be increased only by advertising. Using 
the (14) it is possible show that the advertising effort is constant in time. Therefore, in the absence of a word-of-mouth effect, it becomes optimal to achieve this objective a policy of advertising spending more expensive because it must fill, to ensure the success of event, the absence and the benefits brought about by word of mouth. In other words, in the absence of a word-of-mouth effect, the advertising efforts should actually be spread evenly over time.

An interesting variant of the problem concerns the introduction of a quadratic final penalty $S(x)$.

The function $S(x)$ describes the payoff obtained by the organizers of an event like a concert or a theatre performance. For such events the number of available seats is a crucial parameter. Moreover, it is possible to hypothesize a goodwill threshold $\bar{x}$ such that:

- if the final goodwill exceeds it, i.e. if $x(T)>\bar{x}$, then the demand is greater than the available seats, and there are some unsatisfied consumers;

- if the final goodwill is less than it, i.e. if $x(T)>\bar{x}$ then the demand is less than the available seats, and some tickets remain unsold.

In the first case the organization suffers a loss of reputation, whereas in the latter a loss of revenue. The quadratic penalty function $S(x)$ is a symmetric representation of both these kinds of loss: it is a compromise between the analytical tractability of the problem and a precise description of the economic consequences of observing a demand level above or below the available seats.

\section{Basic Model Advertising for the Launch of a Product}

In this section, the authors study the planning of a pre-launch publicity campaign using the stochastic control theory and some recent results of the stochastic linear quadratic control theory. First of all it must be said that the firm must plan carefully the different marketing actions which it has to take to introduce a new product in the market; in other words, the firm must determine the launch time $T$ (or market entry time), at which the introduction of the product will begin, and must plan the launch advertising campaign on the interval; the sales do not are take into account directly, as they only begin after the launch time $T$, when the launch advertising policy stops. On the other hand, the effects of the advertising policy until the time $T$ on the sales, which will occur later, depend on the goodwil value at $T$ : the greater the goodwill value at $T$, the higher the following sale rate, no matter which advertising policy will be chosen after $T$.

Consequently, the aim of this section is to determine an optimal advertising policy for preparing the launch of a new product, assuming that a firm can control, in the programming interval $[0, T]$, the goodwill evolution of a product through the advertising flow or through some other communication channel and that it wants to maximize the expected utility given by the product goodwill at the (fixed) launch time $T$ and to minimize the total advertising cost.

The firm, namely, wants to drive the goodwill in order to obtain a final demand as close as possible to the congestion threshold minimizing the total ad- 
vertising costs.

The most natural approach to the problem is the description of the goodwill evolution using the stochastic processes theory and this is not new in advertising models. To use the definition of the goodwill is a more promising analytical approach and has considerable intuitive appeal because summarizes the effects of current and past advertising outlays on demand.

The authors present, namely, a linear model of the goodwill evolution under advertising investment. They refer to the concept of goodwill, introduced by Nerlove and Arrow in [14], which resumes the effects of advertising on the demand and whose evolution is described by a linear differential equation which is a generalization of the linear motion equation proposed by Nerlove and Arrow; this last one is a starting point for some practical and theoretical studies in marketing.

Nerlove and Arrow consider the advertising as an investment in a stock (the goodwill $A_{t}$ ), which summarizes the effects of current and past advertising flow $a_{t}$ and assume that the evolution of the goodwill satisfies the first order linear differential equation:

$$
\dot{A}_{t}=-\delta A_{t}+a_{t}
$$

where $a_{t}$ is current advertising expense, $a_{t}$ and $A_{t}$ are understood to be functions of time, and the dot denotes differentiation with respect to time. Equation (15) states that the net investment in goodwill is the difference between the gross investment (current advertising outlay) and the depreciation of the stock of goodwill [14].

If $A_{t}$ is the goodwill level at time $t \in[0, T]$, the organization image, as well as the event features, contribute to affect the initial goodwill of the event, $A_{0}$, which is therefore positive; namely:

$$
A_{0}=A>0 .
$$

In other words, if it is assumed further that current advertising expenditure cannot be negative and that depreciation occurs at a constant proportional rate, $\delta>0$, follows:

$$
\dot{A}_{t}+\delta A_{t}=a_{t}>0
$$

The goodwill decreases spontaneously with decay coefficient $\delta>0$ while, instead, $A_{t}$ is always positive and is sustained by the advertising investment $a_{t}$.

Assuming, then, that the publicity campaign is short enough, it is possible suppose that the consumers do not forget the advertising messages, therefore the goodwill decay is negligible. Hence, the goodwill decay term in the Nerlove and Arrow's model is assumed to be zero.

Consequently, the goodwill evolution, without any advertising flow, can be described by the following linear stochastic differential equation (SDE):

$$
\mathrm{d} A_{t}=-\delta A_{t} \mathrm{~d} t+\sigma_{A} A_{t} \mathrm{~d} W_{t}^{A}
$$


where the parameter $\sigma_{A}$ represents the advertising volatility, $\delta>0$ is the marginal productivities in terms of goodwill while $W_{t}$ is a standard Brownian motion.

In other words, the goodwill is the solution of a controlled linear stochastic differential equation.

The above stochastic differential equation shows that the weight of the word-of-mouth communication is proportional to the actual goodwill and that it affects the goodwill randomly. Actual consumers communicate their product experience randomly, either favorably or unfavorably. The advertising message introduces also an uncertainty source in the system, namely, it has an unforeseeable effect on the goodwill, because either it may not be either completely understood by the consumers, or it may completely meet the taste of the public. In other words, the potential consumers react randomly to advertising because they can be attracted or repelled by the advertising message. This can be described assuming that the goodwill evolution is represented by the following linear stochastic differential equation:

$$
\mathrm{d} A_{t}=\left(a_{t}-\delta A_{t}\right) \mathrm{d} t+\sigma_{A} A_{t} \mathrm{~d} W_{t}^{A}+\sigma_{a} a_{t} \mathrm{~d} W_{t}^{a}
$$

where $W_{t}^{A}, W_{t}^{a}$ are independent Wiener processes, while $\sigma_{a}$ represents the advertising volatility and describes the rate of uncertainty introduced by advertising. Under these assumptions, also the advertising flow becomes a stochastic process and therefore the problem of determining the advertising policy for the product introduction and the launch time can be described using the stochastic control theory; in other words, the problem of determining an advertising policy in order to have an optimal goodwill level at the final time $T$, when the goodwill is subject to a random evolution, is the following optimal control problem:

$$
\begin{gathered}
\underset{a_{t}>0}{\text { Maximize }} E\left(\int_{0}^{T}-c\left(a_{t}\right) \mathrm{d} t+u\left(A_{T}\right)\right) \\
\text { Subject to }\left\{\begin{array}{l}
\mathrm{d} A_{t}=\left(a_{t}-\delta A_{t}\right) \mathrm{d} t+\sigma_{A} A_{t} \mathrm{~d} W_{t}^{A}+\sigma_{a} a_{t} \mathrm{~d} W_{t}^{a} \\
A_{o}=A>0
\end{array}\right.
\end{gathered}
$$

where

- the function $c\left(a_{t}\right)$ represents the cost of advertising that the firm has to sustain in order to obtain an advertising flow $a_{t}$ and, according to literature, it is non-linear, increasing, and convex $\left(c^{\prime} \geq 0, c^{\prime \prime} \geq 0\right)$

- the function $u\left(A_{T}\right)$ represents the utility considered as a prospective profit given by a final level of goodwill $A_{T}$ from the final goodwill and is homogeneous with the cost, it is twice continuously differentiable and increasing $\left(u^{\prime}>0\right)$; in particular, the function $u\left(A_{T}\right)$ describes an estimate of the expected revenue since all the utility obtained by the firm is concentrated at the end of the programming interval

- $\delta>0$ is the decay coefficient

- $A \in R \quad(A>0)$ is a constant which represents the goodwill level at the time 0 , namely the initial value of the goodwill 
- the first diffusion term, $\sigma_{a} A_{t} \mathrm{~d} W_{t}^{a}$, accounts for the word-of mouth communication

- the parameter $\sigma_{A} \geq 0$ is the advertising volatility and describes the power of this effect

- the second diffusion term, $\sigma_{a} a_{t} \mathrm{~d} W_{t}^{a}$, accounts for the lure/repulsion effect of advertising

- the parameter $\sigma_{a} \geq 0$ is the communication effectiveness volatility.

It is possible note that, although the goodwill is assumed to be positive at the initial time, it may as well be negative at any time $t>0$. Furthermore, may be single out three special situations which are associated with the following choices of diffusion coefficients:

$\sigma_{\mathrm{A}}=\sigma_{a}=0$, i.e. the deterministic case with motion Equation (1);

$\sigma_{A}>0, \sigma_{a}=0$, i.e. the case in which the word-of-mouth phenomenon is the unique cause of randomness;

$\sigma_{A}=0, \sigma_{a}>0$, i.e. the case in which the advertising lure/repulsion effect is the unique source of randomness.

The model considered assumes that the demand depended on the goodwill only, namely essentially on the firm marketing strategies. In practice, the demand depends also on the competitors actions. Hence, a good model should account for the competition among the different agents acting in the market. A firm profit problem is part of a dynamic game, where firms are the players, each with its own objective and available strategies.

\section{Remarks and Future Perspectives for Research}

In this paper have been identified and discussed the optimal advertising policies to follows for an event, that takes place at a given and future period of time, in order to be able to place on the market all or the vast majority of tickets and ensure, therefore, the success of the event. The ticket sales are driven by the advertising made by the organizers as well as by the word-of-mouth effect. In other words, the seat security or the location of the ticket is guaranteed, as it is said before, by two elements: the advertising implemented by the organizers (who can use a variety of communication tools: internet, print media, TV channels etc.) as well as by word of mouth (that becomes a great channel for conveying information and at the same time guarantees a decrease in advertising costs). In both cases, it is assumed that the price of a ticket is fixed.

Clearly are of interest some conjectures. For instance, an interesting problem would be to study the case where the price can be discounted at a certain instant of time, with the intention of boosting the sales. The potential consumers would then decide between having a guaranteed a seat at a higher price and paying a reduced price for a seat the availability of which is uncertain. Then, the question, which leads always to a problem of optimization, becomes when to discount the ticket price and with what amount.

It is of interest to test also the following conjecture. As has been said before, 
the results of the word-of-mouth effect in advertising efforts, mainly concentrated at the beginning of the planning period, has been obtained in the absence of discounting. In fact, the planning horizon, considered in the previous sections, is typically short and hence there is no need for discounting. The authors believe that this assumption is fully justified in context of a short planning period but they think that the introduction of positive discounting will causes a shift of advertising efforts to later time instances, so that the word-of mouth effect will be modified.

\section{Conclusions}

The recent mathematical developments in this area represent a very useful tool to study some interesting economic problems and different special instances of the problem.

The authors hope to have provided, through this paper, a small step in research on the advertising policies and also useful and interesting research directions; they believe, however, that is still needed a significant research and hope that this paper will stimulate extensions and practical applications. It will be interesting to see how future research will offer, in the future, some valid ideas.

\section{References}

[1] Robinson, B. and Lakhani, C. (1975) Dynamic Price Models for New-Product Planning. Management Science, 21, 1113-1122. https://doi.org/10.1287/mnsc.21.10.1113

[2] Feichtinger, G., Hartl, R.F. and Sethi, S.P. (1994) Dynamic Optimal Control Models in Advertising: Recent Developments. Management Science, 40, 195-226. https://doi.org/10.1287/mnsc.40.2.195

[3] Ferrentino, R. and Boniello, C. (2015) Communication and Advertising: A Stochastic Approach. Journal of Analysis and Applications, 13, 1-13.

[4] Jørgensen, S. and Zaccour, G. (2004) Differential Games in Marketing. Kluwer Academic Publishers, Boston, MA. https://doi.org/10.1007/978-1-4419-8929-1

[5] Ozga, S. (1960) Imperfect Markets through Lack of Knowledge. Quarterly Journal of Economics, 74, 29-52. https://doi.org/10.2307/1884132

[6] Gould, J.P. (1970) Diffusion Processes and Optimal Advertising Policy In: Phelps, E.S., et al., Ed., Microeconomic Foundation of Employment and Inflation Theory, W.W. Norton, New York, 338-368.

[7] Sethi, S.P. (1979) Optimal Advertising Policy with the Contagion Model. Journal of Optimization Theory and Applications, 29, 615-627. https://doi.org/10.1007/BF00934454

[8] Sethi, S.P. (1974) Some Explanatory Remarks on the Optimal Control of the Vidale-Wolfe Advertising Model. Operations Research, 22, 1119-1120. https://doi.org/10.1287/opre.22.5.1119

[9] Teng, J.T. and Thompson, G.L. (1983) Oligopoly Models for Optimal Advertising When Production Costs Obey a Learning Curve. Management Science, 29, 1087-1101. https://doi.org/10.1287/mnsc.29.9.1087

[10] Dockner, E.J. and Jørgensen, S. (1998) Optimal Advertising Policies for Diffusion 
Models of New Product Innovation in Monopolistic Situations. Management Science, 34, 119-130. https://doi.org/10.1287/mnsc.34.1.119

[11] Dockner, E.J. and Jørgensen, S. (1992) New Product Advertising in Dynamic Oligopolies. Zeitschrift für Operations Research, 36, 459-473.

[12] Horsky, D. and Mate, K. (1998) Dynamic Advertising Strategies of Competing Durable Goods Producers. Marketing Science, 7, 356-367.

https://doi.org/10.1287/mksc.7.4.356

[13] Buratto, A., Grosset, L. and Viscolani, B. (2005) Linear Model and Advertising. Rendiconti per gli studi Economici e Quantitativi, 107-120.

[14] Nerlove, M. and Arrow, J.K. (1962) Optimal Advertising Policy under Dynamic Conditions. Economica, 29, 129-142.

[15] Shone, A. and Parry, B. (2004) Successful Event Management.

[16] Godes, D. and Mayzlin, D. (2004) Using Online Conversations to Study Word of Mouth Communication. Marketing Science, 23, 545-560. https://doi.org/10.1287/mksc.1040.0071

[17] Seierstad, A. and Sydsæter, K. (1987) Optimal Control Theory with Economic Applications. North-Holland, Amsterdam. 